\title{
$\mathbf{J}|\mathbf{A}| \mathbf{C} \mid \mathbf{S}$ \\ COMMUNICATIONS
}

Published on Web 11/22/2002

\section{Synthesis of Silver Nanodisks Using Polystyrene Mesospheres as Templates}

\author{
Encai Hao, K. Lance Kelly, Joseph T. Hupp, ${ }^{*}$ and George C. Schatz* \\ Department of Chemistry, Northwestern University, 2145 Sheridan Road, Evanston, Illinois 60208
}

Received August 29, 2002

The remarkable size and shape dependence of physical, optical, and electronic properties of metal and semiconductor nanocrystals make them compelling components of modern materials chemistry. ${ }^{1-3}$ When spherical metal particles are transformed into nanoscale rods or triangular prisms, the surface plasmon resonances are strongly affected, typically red-shifting and even splitting into distinctive dipole and quadrupole plasmon modes. ${ }^{4}$ Colloid chemists have recently made much progress in preparing monodispersed spherical nanocrystals via ambient temperature wet-chemical routes or organometallic methods. ${ }^{2}$ Although the synthesis of spherical nanocrystals is well developed for many materials, systematic manipulation of the shapes of nanocrystals is a significant challenge. Recent work devoted to making nonspherical nanocrystals via anisotropic inorganic nanocrystal growth in liquid media (including electrochemical growth) has yielded Au rods, ${ }^{5} \mathrm{BaCrO}_{4}$ rods, ${ }^{6} \mathrm{TiO}_{2}$ rods, ${ }^{7} \mathrm{Ag}$ rods, ${ }^{8} \mathrm{Pt}$ cubes and pyramids, ${ }^{9}$ and rod-, arrow-, teardrop-, and tetrapod-shaped CdSe nanocrystals. ${ }^{10,11}$ Fabrication of these materials is useful not only for understanding fundamental phenomena such as quantum confinement but also for obtaining "building blocks" featuring optical and electronic properties that are desirable for advanced applications in photovoltaic solar cells, light-emitting diodes, and biological diagnostics. ${ }^{4,12,13}$

In contrast to nanorods, nanodisks and nanoprisms such as triangles are less well-known. Although a few strategies do exist for synthesizing Co disks, ${ }^{14} \mathrm{Ag}$ disks, ${ }^{15}$ and Ag triangle nanoprisms, ${ }^{4,16}$ there is great interest in developing new methods for making nanoparticles with control over shape and size. Herein, we report a new synthesis of disk-shaped Ag nanoparticles using polystyrene spheres as templates.

An aqueous colloidal suspension of carboxylate-functionalized polystyrene (PS) spheres was purchased from Interfacial Dynamics Corporation. Fresh $\mathrm{Ag}$ solution containing $10 \mathrm{wt} \% \mathrm{AgNO}_{3}$ and $6 \%$ ammonium hydroxide was prepared. Briefly, $160 \mu \mathrm{L}$ of $110-$ $\mathrm{nm}$ diameter PS spheres and $75 \mu \mathrm{L}$ of $\mathrm{Ag}$ solution were added to $60 \mathrm{~mL}$ of somewhat aged $N, N$-dimethylformamide (DMF). Then, the solution was heated with stirring on a hotplate. Within 8-12 min, the solution color changed from yellow through red to purple, after which the temperature was quickly reduced via a cold-water bath. The product was purified by gradient centrifugation and then kept at $4{ }^{\circ} \mathrm{C}$.

A rich variety of recipes are now available for making $\mathrm{Ag}$ nanoparticles in solution. DMF is one of the standard organic compounds used both as solvent and reducing agent. The reduction of $\mathrm{Ag}^{+}$ions in DMF can take place at room temperature, but the reaction rate is markedly increased at higher temperatures. It is also known that DMF slightly decomposes to a more easily oxidized amine upon aging or upon catalytic decomposition with solid base. The availability of the amine evidently accelerates the reduction of silver ions. (We found that dialkylamines are ineffective as

* To whom correspondence should be addressed. E-mail: jthupp@ chem.nwu.edu and schatz@chem.nwu.edu.

15182 a J. AM. CHEM. SOC. 2002, 124, 15182-15183

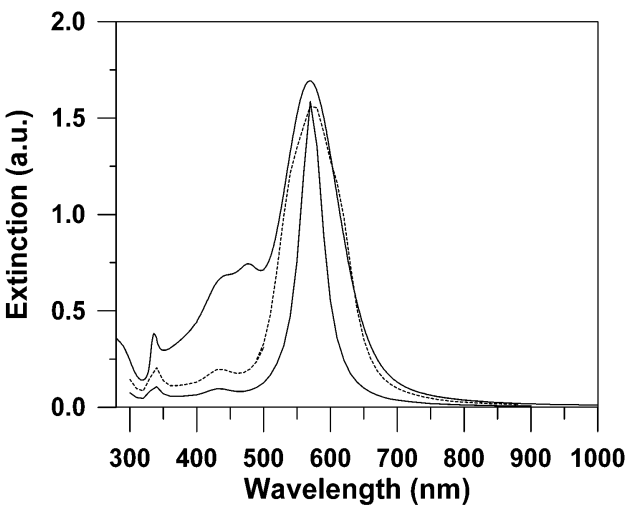

Figure 1. Solid line (upper) is the UV-vis spectrum of $\mathrm{Ag} / \mathrm{PS}$ in $\mathrm{DMF}$. Solid line (bottom) is the DDA simulation of the orientation averaged extinction efficiency spectrum of Ag disk (single size) in DMF. Dashed line is the weighted-average DDA simulation for a distribution of three sizes (TEM).

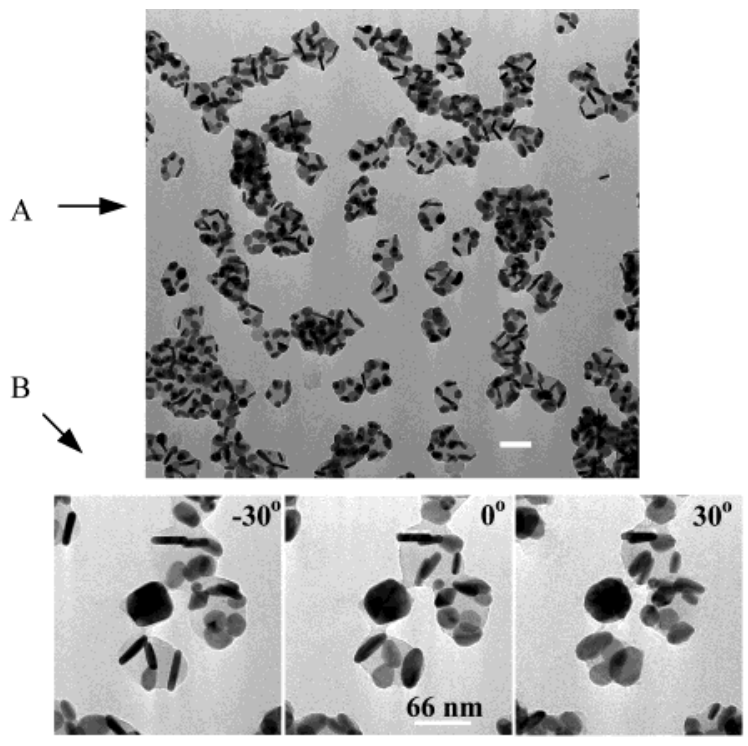

Figure 2. (A) TEM image of a fresh Ag/PS sample. The tool bar is 100 $\mathrm{nm}$. (B) TEM images of $\mathrm{Ag} / \mathrm{PS}$, tilting the sample plane from $-30^{\circ}$ through $0^{\circ}$ to $+30^{\circ}$.

additives to fresh DMF but that added hydroxyl-terminated alkylamines do facilitate nanoparticle formation.) Colloidal Ag particles prepared using DMF are usually spherical with a plasmon absorption band centered at $415 \mathrm{~nm} .{ }^{17,18}$ When colloidal polystyrene is included in the preparation, a set of color changes is observed. The final UV-vis spectrum (Figure 1) shows bands at $\lambda_{\max }=340$ (weak) and 574 (strong), with other peaks between 430 and 470 nm.

Transmission electron microscopy (TEM, Figure 2A) of a fresh sample shows individual and aggregated polystyrene particles 


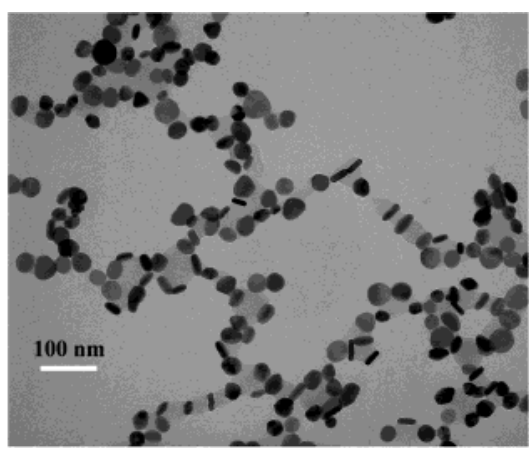

Figure 3. TEM image of $\mathrm{Ag} / \mathrm{PS}$ sample after aging 1 month.

inhomogeneously covered by silver disks that are randomly oriented on the particle surface. The disk shape was verified by tilting the TEM sample plane from $-30^{\circ}$ through $0^{\circ}$ to $+30^{\circ}$ (see Figure $2 \mathrm{~B}$ ), revealing the three-dimensional structure of the nanoparticles. At this stage almost no $\mathrm{Ag}$ particles unattached to polystyrene are seen. The geometry of the attached particles suggests that they grow anisotropically on the surface of PS spheres. The presence of edgeoriented disks, appearing as rodlike shapes in the TEM images, allows measurement of their thickness $(9.0 \pm 1.0 \mathrm{~nm})$. The average disk diameter is $36 \pm 8 \mathrm{~nm}$.

The TEM image in Figure 3 shows that after one month of aging, most of the PS mesospheres are dissolved, yielding large quantities of unattached Ag nanodisks. The ability to dissolve the template particles without destroying the disk structures could be important for advanced applications. From Figure 3, the fraction of $\mathrm{Ag}$ particles present as disks exceeds $90 \%$; only small quantities with other shapes, for example, triangles, have been observed.

It is worth mentioning that, during the initial synthesis of $\mathrm{Ag}$ nanodisks, because the reaction requires only several minutes the polystyrene particles are not destroyed. Under surfactant-free conditions, the particles appear to play an essential templating role. We postulate electrostatic binding (adsorption) of $\mathrm{Ag}$ ions by surface carboxyl groups of the mesospheres. Upon reduction, the adsorbed Ag atoms presumably serve to nucleate metal-particle formation, a process that is accelerated by temperature elevation. Once nucleation is initiated, the mesospheres may block growth in one direction, effectively templating growth in another (lateral) direction. The available microscopy also suggests that anisotropic growth occurs between PS mesospheres, leading to aggregation of the templating units. Yet to be determined is the effect, if any, of mesosphere curvature on Ag nanoparticle size and morphology. Clearly, however, the curvature of 110-nm spheres does not result in simple polystyrene core/Ag shell structures. ${ }^{19}$

Consistent with Mie theory, ${ }^{20}$ spherical Ag nanoparticles in DMF exhibit a single-surface plasmon resonance centered at $415 \mathrm{~nm}$. The Ag nanodisks presented here exhibit optical properties that are remarkably different from those of spherical Ag nanoparticles. They are more similar to those of Ag triangle nanoprisms. To gain insight into the optical properties, we have solved Maxwell's equations for light interacting with $\mathrm{Ag}$ nanodisks using the discrete dipole approximation (DDA). ${ }^{21} \mathrm{We}$ consider disks with dimensions given by TEM. For the Ag disk nanoprisms, the induced polarizations lead to three bands that qualitatively match the experimental results shown in Figure 1. Analysis of the induced field shows that the 574-nm peak is the in-plane dipole plasmon resonance, the 340$\mathrm{nm}$ peak is the out-of-plane quadrupole resonance, and the weak 430-nm peak is the out-of-plane dipole resonance. Only the 574$\mathrm{nm}$ peak is found to be very sensitive to the disk thickness and dimensions. One explanation for the band around $470 \mathrm{~nm}$ is the presence of trace quantities of strongly absorbing triangular prisms. A strong in-plane quadrupole resonance has previously been seen at this wavelength for appropriately sized $\mathrm{Ag}$ triangles, ${ }^{4}$ but for the nanodisks, quadrupole resonances are found in the DDA calculations to be less intense. This behavior is to be expected, as the quadrupole intensity increases more quickly with particle size than does the dipole intensity. It's also possible that aggregation of the disks is important as recently described for gold particles. ${ }^{22}$

In summary, we report the synthesis of $\mathrm{Ag}$ nanodisks using polystyrene mesospheres as templates. The disks are quite different from those prepared using other methods, ${ }^{15}$ and they exhibit an intense electronic spectrum that differs markedly from those for spheres, however, qualitatively matching DDA theory calculations. The spectral differences could prove to be of considerable importance in frequency-doubling ${ }^{23}$ and other nonlinear processes that depend on resonant or near-resonant interactions. The communications-relevant 1550-to-775 nm conversion, for example, could be easier to accomplish with disks than with spheres because of near-resonance at the doubling wavelength.

Acknowledgment. We gratefully acknowledge support from the National Science Foundation through the Northwestern Materials Research Center (MRSEC). E.H. thanks Mr. Rongchao Jin and Dr. Ryan Sun (Northwestern University) for valuable discussions.

\section{References}

(1) Alivisatos, A. P. J. Phys. Chem. 1996, 100, 13226

(2) Murray, C. B.; Norris, D. J.; Bawendi, M. G. J. Am. Chem. Soc. 1993, 115,8706

(3) Lieber, C. M. Solid State Commun. 1998, 107, 607.

(4) Jin, R.; Cao, Y.; Mirkin, C. A.; Kelly, K. L.; Schatz, G. C.; Zheng, J. G. Science 2001, 294, 1901

(5) Yu, Y.-Y.; Chang, S.-S.; Lee, C.-L.; Wang, C. R. C. J. Phys. Chem. B 1997, 101, 6661

(6) Li, M.; Schnablegger, H.; Mann, S. Nature 1999, 402, 393.

(7) Chemseddine, A.; Moritz, T. Eur. Inorg. Chem. 1999, 235.

(8) Jana, N. R.; Gearheart, L.; Murphy, C. J. Chem. Commun. 2001, 617.

(9) Ahmadi, T. S.; Wang, Z. L.; Green, T. C.; Henglein, A.; El-Sayed, M. A Science 1996, 272, 1924

(10) Manna, L.; Scher, E. C.; Alivisatos, A. P. J. Am. Chem. Soc. 2000, 122, 12700 .

(11) Peng, X.; Manna, L.; Yang, W.; Wickham, J.; Scher, E.; Kadavanich, A.; Alivisatos, A. P. Nature 2000, 404, 59

(12) Huynh, W. U.; Dittmer, J. J.; Alivisatos, A. P. Science 2002, 295, 2425

(13) McConnell, W. P.; Novak, J. P.; Brousseau, L. C., III; Fuierer, R. R.; Tenent, R. C.; Feldheim, D. L. J. Phys. Chem. B 2000, 104, 8925.

(14) Puntes, V. F.; Krishnan, K. M.; Alivisatos, A. P. Science 2001, 291, 2115.

(15) Chen, S.; Fan, Z.; Carroll, D. L. J. Phys. Chem. B 2002, 106, 10777.

(16) Chen, S.; Carroll, D. L. Nano Lett. 2002, 2, 1003.

(17) Pastoriza-Santos, I.; Liz-Marzán, L. M. Langmuir 1999, 15, 948.

(18) Pastoriza-Santos, I.; Koktysh, D. S.; Mamedov, A. A.; Giersig, M.; Kotov, N. A.; Liz-Marzán, L. M. Langmuir 2000, 16, 2731.

(19) Jackson, J. B.; Halas, N. J. J. Phys. Chem. B 2001, 105, 2743.

(20) Bohren, C. F.; Huffman, D. R. Absorption and Scattering of Light by Small Particles; Wiley-Interscience: New York, 1983.

(21) Yang, W. H.; Schatz, G. C.; Van Duyne, R. P. J. Chem. Phys. 1995, 103, 869.

(22) Norman, T. J., Jr.; Grant, C. D.; Magana, D.; Zhang, J. Z.; Liu, J.; Cao, D.; Bridges, F.; Van B. A. J. Phys. Chem. B 2002, 106, 7005.

(23) Hao, E. C.; Schatz, G. C.; Johnson, R. C.; Hupp, J. T. J. Chem. Phys. 2002, 117, 5963.

JA028336R 